\title{
Measurement of Brain Metabolites in Patients with type 2 Diabetes and Major Depression Using Proton Magnetic Resonance Spectroscopy
}

\author{
Olusola Ajilore*,', Ebrahim Haroon', Senthil Kumaran², Christine Darwin ${ }^{3}$, Nader Binesh², Jim Mintz', \\ Jacqueline Miller', M Albert Thomas ${ }^{2}$ and Anand Kumar' \\ 'Department of Psychiatry, UCLA Semel Institute, Los Angeles, CA, USA; ${ }^{2}$ Department of Radiology, UCLA, Los Angeles, CA, USA; ${ }^{3}$ Department \\ of Clinical Epidemiology and Preventative Medicine, UCLA, Los Angeles, CA, USA
}

\begin{abstract}
Type 2 diabetes and major depression are disorders that are mutual risk factors and may share similar pathophysiological mechanisms. To further understand these shared mechanisms, the purpose of our study was to examine the biochemical basis of depression in patients with type 2 diabetes using proton MRS. Patients with type 2 diabetes and major depression $(n=20)$ were scanned along with patients with diabetes alone $(n=24)$ and healthy controls $(n=21)$ on a I.5T MRI/MRS scanner. Voxels were placed bilaterally in dorsolateral white matter and the subcortical nuclei region, both areas important in the circuitry of late-life depression. Absolute values of myoinositol, creatine, $\mathrm{N}$-acetyl aspartate, glutamate, glutamine, and choline corrected for CSF were measured using the LC-Model algorithm. Glutamine and glutamate concentrations in depressed diabetic patients were significantly lower $(p<0.00 \mathrm{I})$ in the subcortical regions as compared to healthy and diabetic control subjects. Myo-inositol concentrations were significantly increased $(p<0.05)$ in diabetic control subjects and depressed diabetic patients in frontal white matter as compared to healthy controls. These findings have broad implications and suggest that alterations in glutamate and glutamine levels in subcortical regions along with white matter changes in myo-inositol provide important neurobiological substrates of mood disorders.

Neuropsychopharmacology (2007) 32, 1224-1231. doi: I0.1038/sj.npp. I 301248; published online 20 December 2006
\end{abstract}

Keywords: major depression; diabetes; myo-inositol; glutamate; spectroscopy

\section{INTRODUCTION}

Diabetes is a major health problem affecting approximately 18 million Americans (Centers for Disease Control and Prevention, 2004). It is a growing crisis that has devastating complications including heart disease, peripheral neuropathy, renal disease, and retinopathy. Diabetes is also associated with an increased risk of developing major depression. According to a literature review by Gavard et al (1993), the prevalence of major depression in a sample diabetic population in controlled studies was $8.5-27.3 \%$ (mean prevalence of $14.0 \%$ ). This was estimated to be up to three times the prevalence of major depression in the general US adult population. Diabetes and major depression are mutual risk factors with diabetic patients more likely to develop major depression with an odds ratio of 2.5 according to one study (Palinkas et al, 2004) and depressed patients being more likely to develop type 2

*Correspondence: Dr $\bigcirc$ Ajilore, Department of Psychiatry, UCLA Semel Institute, 760 Westwood Plaza, Los Angeles, CA 90024, USA, Tel: + | 310825 5523, Fax: + | 3107949011 ,

E-mail: oajilore@mednet.ucla.edu.

Received 25 January 2006; revised 3 August 2006; accepted 4 August 2006 diabetes with an estimated relative risk of 2.2 according to another study (Eaton et al, 1996). Depression also has a significant impact on the course of diabetes leading to higher rates of hyperglycemia and diabetic complications (de Groot et al, 2001; Lustman and Clouse, 2005).

The link between diabetes and major depression is multifactorial. However, one of the elements common to both disorders, particularly with regards to late-life major depression, is the role of vascular disease. Previous studies have looked at the role of vascular disease in both disorders. It has been shown that patients with major depression and diabetes have a 1.5- to 2-fold greater chance of having three or more cardiovascular risk factors as compared to diabetic patients without depression (Katon et al, 2004). In addition, in late-life depression, disruptions of frontal-subcortical circuits have been implicated due to the interplay of vascular disease and white-matter abnormalities (Kumar and Cook, 2002). Thus, brain changes associated with type 2 diabetes might reveal a common pathophysiological pathway involving vascularly mediated impairment of whitematter tracts in frontal-subcortical circuits.

A method useful in determining the biochemical perturbations associated with this type of impairment is proton magnetic resonance spectroscopy (MRS). Proton MRS has 
facilitated the study of NAA, choline, myo-inositol, and Glx (a composite in vivo signal of glutamate and glutamine) in focal brain regions. Previous papers that have used MRS to probe the biochemical changes in the brain induced by major depression and diabetes have shown relatively consistent patterns particular to each disease. Studies that have used MRS to examine changes in brain metabolites related to diabetes have consistently shown increases in myo-inositol (Kreis and Ross, 1992; Geissler et al, 2003; van der Graaf et al, 2004; Makimattila et al, 2004). Use of MRS to study the biochemical changes associated with major depression has shown a number of findings over the past few years. These include increases in choline, myo-inositol, and decreases in $n$-acetyl aspartate and glutamate associated with major depression (Kumar et al, 2002; Vythilingam et al, 2003; Auer et al, 2000; Rosenberg et al, 2005). These metabolic changes associated with major depression have been shown to correlate with electroconvulsive and antidepressant treatment as well. An increase in amygdalar region $n$-acetyl aspartate was seen in depressed patients who exhibited a positive response to electroconvulsive therapy (Michael et al, 2003a-c). In addition, Glx concentrations in left dorsal lateral prefrontal cortex were negatively correlated with severity of depression and increased significantly after electroconvulsive treatment (Michael et al, 2003a-c). One study showed an increase in glutamate levels associated with major depression, but unlike previous studies, the region of interest was in the occipital cortex (Sanacora et al, 2004). Other brain regions have been studied as well using MRS in major depression. For example, MRS of the mesial temporal lobe of depressed patients demonstrated significant increases in choline to creatine ratios as compared to controls (Mervaala et al, 2000).

The purpose of our study was to examine the metabolites associated with depression in patients with type 2 diabetes using proton MRS. We were interested in examining the role of frontal and subcortical regions implicated in the pathophysiology of mood disorders, namely the dorsolateral prefrontal cortex and the subcortical nuclei region, including the caudate nucleus. More specifically, we were interested in estimating the levels of $n$-acetylaspartate (NAA), choline, glutamate, glutamine, and myo-inositol bilaterally in dorsolateral white matter and the subcortical nuclei region. We hypothesized that patients with type 2 diabetes and major depression would have lower levels of NAA, associated with higher levels of myo-inositol and choline when compared to healthy control subjects. We also hypothesized that there would be lower glutamate concentrations in patients with type 2 diabetes and major depression as compared to healthy control subjects. In addition, we hypothesized that patients with diabetes and without depression would show metabolite levels that were in between our healthy group and our sample of patients with depression and diabetes.

\section{MATERIALS AND METHODS}

\section{Subjects}

We enrolled 47 patients between the ages of 30 and 80 , diagnosed with type 2 diabetes by their primary care physicians. Of these, 20 met DSM-IV criteria for major depressive disorder, and 24 denied a history of or current depression and were enrolled as diabetic controls. Three other patients were diagnosed with minor depression and excluded from the present analyses. Patients were recruited from three outpatient clinical sites: Gonda Diabetes Center at UCLA, UCLA Division of Endocrinology (Santa Monica), and a satellite diabetes clinic (Alhambra, CA). Twenty-one control participants were recruited through newspaper advertisements circulating in Los Angeles, CA.

All subjects were given a structured clinical interview (Structured Clinical Interview for DSM-IV) assessed by board-certified psychiatrists (AK and EH) Depressed patients received a score of 15 or higher (mean score $20.7 \pm 3.78)$ on the Hamilton Rating Scale for Depression (Hamilton, 1967) and none of them had any psychotic features clinically. None of the patients had either a history or mental status consistent with progressive cognitive decline and scored 24 or above on the Mini-Mental State Exam (MMSE, Folstein et al, 1975). All patients with depression were free of antidepressant medications for at least 2 weeks prior to the study. Many of them were drugnaïve. Five of the 20 subjects reported having an episode of depression prior to the onset of diabetes. Diabetic patients were on varying combinations of oral hypoglycemic agents and insulin for blood sugar control. Out of 24 diabetic control subjects, 16 were taking oral hypoglycemics only, two were on insulin alone, and five were on a combination of the two. Out of 20 depressed diabetic subjects: 10 were on oral hypoglemic agents, one was on insulin alone, and six were on a combination of the two. Two depressed diabetic subjects and one diabetic control subject were on no medications for glucose control. Exclusion criteria for the present study included the following: Dementia, central nervous system diseases, unstable medical illnesses, other Axis I disorders (including bipolar disorder and psychotic disorders), drug or alcohol dependence, head trauma or an MMSE score of less than 24. Health status and medical comorbidities were assessed using the Cumulative Illness Rating Scale for Geriatrics (CIRS-G, Linn et al, 1968) and the Cerebrovascular Risk Factor Scale (CVRF, D'Agostino et al, 1994). To determine glycemic control, hemoglobin A1c (Hgb A1c) levels were measured for all subjects. Both diabetic groups had significantly higher ratings on CIRS and CVRF, as well as higher levels of Hgb Alc compared to healthy controls. While depressed diabetic subjects had higher ratings and levels as compared to diabetic control subjects, there was no significant difference between diabetic groups. Mean values for these clinical measures across subject groups are summarized in Table 1.

\section{MRS Acquisition}

A GE 1.5T MRI/MRS scanner (General Electric Medical Systems, Waukesha, WI) operating on Lx platform was used along with a standard birdcage quadrature head coil for transmission/reception. Four voxels of $4 \mathrm{ml}$ $(2 \mathrm{ml} \times 2 \mathrm{ml} \times 1 \mathrm{ml})$ size were localized using PRESS sequence on bilateral subcortical nuclei and bilateral dorsolateral prefrontal white matter regions. Water suppression was achieved with the help of CHESS pulses. Numerically optimized Shinnar-Le Roux (SLR) rf pulses were used for PRESS $\left(90^{\circ}, 180^{\circ}, 180^{\circ}\right)$ and CHESS $\left(90^{\circ}, 90^{\circ}, 90^{\circ}\right)$. 
Table I Clinical Measures across Subject Groups

\begin{tabular}{|c|c|c|c|c|c|c|}
\hline & Healthy controls $(n=2 I)$ & Diabetic controls $(n=24)$ & Depressed diabetic $(n=20)$ & $\mathbf{F}$ & df & $p$-value \\
\hline Age & $55.67 \pm 9.8674$ & $58.13 \pm 9.236$ & $56.55 \pm 10.2208$ & 0.3741 & 2 & 0.696 \\
\hline \multicolumn{7}{|l|}{ Gender } \\
\hline$\%$ Male $\%$ & $23.81-$ & $29.17-$ & $25.0-$ & & & \\
\hline \% Female & $76.19-$ & $70.83-$ & $75.0-$ & $\chi^{2}=0.186$ & - & 0.91 \\
\hline CIRS米米 & $2.81 \pm 2.385$ & $5.50 \pm 3.329$ & $6.80 \pm 3.140$ & 9.5961 & 2 & 0.00022 \\
\hline CVRF**** & $4.81 \pm 4.4237$ & $10.96 \pm 4.261$ & $|1.50 \pm 5.38|$ & | 3.3576 & 2 & $<0.0001$ \\
\hline Hgb Alc****** & $5.31 \pm 0.365$ & $7.13 \pm 1.064$ & $7.45 \pm 1.542$ & 23.62261 & 2 & $<0.0001$ \\
\hline
\end{tabular}

Data are presented as means \pm SD.

MMSE, Mini-Mental Status Exam; CIRS, Cumulative IIIness Rating Scale; CVRF, Cerebrovascular Risk Factor Scale; Hgb Alc, Hemoglobin A Ic. Healthy controls significantly lower than both diabetic subject groups on CIRS, CVRF, and Hgb AIc measures.

**** $P<0.001$.

Following parameters were used for spectral acquisition: $\mathrm{TR}=3 \mathrm{~s}, \mathrm{TE}=30 \mathrm{~ms}$, and $\mathrm{NEX}=128$. Four unsuppressed water FIDs were also acquired from each location for eddy current compensation and phase correction.

\section{MRS Post-Processing}

The MRS raw files were transferred to an offline SGI O2 workstation (Silicon Graphics Inc, Sunnyvale, CA). The raw files were processed using LC-Model package. The water suppressed time domain data were analyzed between 1.0 and $4.0 \mathrm{ppm}$ without further $\mathrm{T} 1$ and $\mathrm{T} 2$ correction. The basis set provided by the vendor was used and then scaled to a consistent transmitter gain (TG). Absolute values of $\mathrm{N}$ acetyl aspartate (NAA), creatine ( $\mathrm{Cr}$ ), glutamate/glutamine $(\mathrm{Glx})$, glutamate $(\mathrm{Glu})$, glutamine $(\mathrm{Gln})$, choline $(\mathrm{Ch})$, and myo-inositol (MI) are reported in millimolar uncorrected for T1 and T2 relaxation. A sample spectrum is shown in Figure 1. The absolute metabolite values were further corrected for cerebrospinal fluid (CSF) by segmenting the 3D SPGR coronal MR images in to Gray (GM), White (WM), and CSF (Kollokia, 1996). There were no significant differences in the cerebrospinal fluid or gray and white matter composition of the voxels between subject groups (Tables 2 and 3).

\section{Anatomical Landmarks}

The axial slice showing the most anterior extent of the anterior margin of the genu of the corpus callosum was chosen as the reference image on which to center the voxels for both locations (Kumar et al, 2002). This was determined by placing a cursor at the anterior margin of the genu in the midline, on several axial slices through the genu. The slice showing the most anterior location of the anterior margin of the genu was then chosen as the reference slice. This anatomical landmark was chosen because it is an identifiable single location, which helps standardize voxel placement, despite variations in scanning angle. Other potential single location sites in the brain are at a greater distance from the areas of interest, and therefore the potential error,

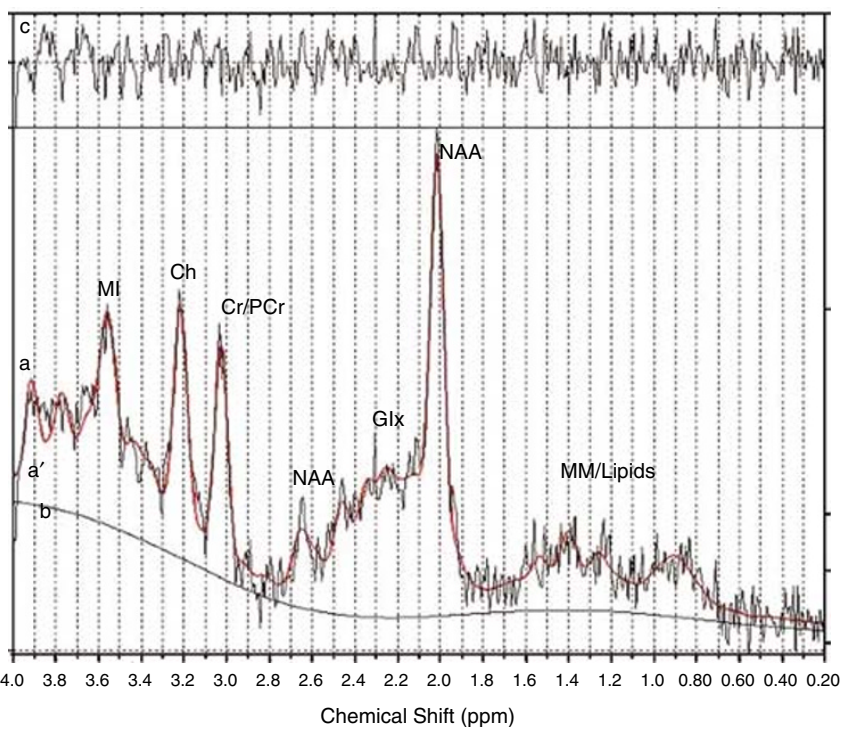

Figure I A water-suppressed IH MR spectrum of a 62-year-old patient with diabetic depression using TR/TE $=3 \mathrm{~s} / 30 \mathrm{~ms}, 128$ averages and a voxel size of $4 \mathrm{ml}$. (a') experimental and (a) LC-Model fitted spectrum, (b) baseline, and (c) noise predicted by the curve-fitting algorithm.

due to variation in scanning angle, is greater. This slice corresponds to a level $10 \mathrm{~mm}$ superior to the AC-PC line, though variations in head size will subtly impact the precise location.

Dorsolateral prefrontal cortex. The medial border of the voxel is placed contiguous to the gray-white juncture on the medial side of the hemisphere using the same reference slice as above. The posterior boundary of the voxel is placed immediately adjacent to the anterior margin of the frontal horn of the lateral ventricle.

Subcortical voxel. The mesial border of the voxel is at the junction of the head of the caudate nucleus and the lateral ventricle. The posterior boundary does not extend below the inferior tip of frontal horn of the lateral ventricle (Figure 2). 
Table 2 White Matter (WM), Gray Matter (GM), and Cerebrospinal Fluid (CSF) Composition of MRS Voxels in the Subcortical Nuclei Region of Healthy Controls (HC), Diabetic Subjects (DC), and Depressed Diabetic Subjects (DD)

Subcortical voxels

\begin{tabular}{|c|c|c|c|c|c|c|}
\hline & \multicolumn{3}{|c|}{ Left (\%) } & \multicolumn{3}{|c|}{ Right (\%) } \\
\hline & WM & GM & CSF & WM & $\mathbf{G M}$ & CSF \\
\hline DC & $37.01 \pm 12.59$ & $49.10 \pm 9.74$ & $13.17 \pm 7.35$ & $59.43 \pm 10.26$ & $37.37 \pm 8.80$ & $3.07 \pm 2.73$ \\
\hline DD & $35.74 \pm 13.39$ & $48.02 \pm 9.62$ & $15.67 \pm 12.22$ & $64.56 \pm 12.66$ & $31.96 \pm 10.05$ & $3.44 \pm 4.81$ \\
\hline
\end{tabular}

Percentages shown as means \pm SD.

HC, healthy controls $(n=21)$; DC, diabetic controls $(n=24)$; DD, depressed diabetic subjects $(n=20)$.

Table 3 White Matter (WM), Gray Matter (GM), and Cerebrospinal Fluid (CSF) Composition of MRS Voxels in Frontal WM of HC, DC, and DD subjects

Frontal white matter

Left (\%)

\begin{tabular}{|c|c|c|c|c|c|c|}
\hline & & \\
\hline & WM & $\mathbf{G M}$ & CSF & WM & $\mathbf{G M}$ & CSF \\
\hline $\mathrm{HC}$ & $82.83 \pm 9.17$ & $16.82 \pm 8.90$ & $0.34 \pm 0.54$ & $84.30 \pm 6.57$ & $15.39 \pm 6.32$ & $0.31 \pm 0.41$ \\
\hline DC & $83.13 \pm 7.16$ & $16.50 \pm 6.93$ & $0.37 \pm 0.35$ & $86.59 \pm 6.22$ & $13.06 \pm 6.02$ & $0.35 \pm 0.41$ \\
\hline DD & $82.05 \pm 9.08$ & $17.38 \pm 8.52$ & $0.57 \pm 0.78$ & $84.28 \pm 7.63$ & $15.40 \pm 7.36$ & $0.32 \pm 0.53$ \\
\hline
\end{tabular}

Percentages shown as means \pm SD.

HC, healthy controls $(n=21)$, DC, diabetic controls $(n=24)$; DD, depressed diabetic subjects $(n=20)$.

Note that voxels placed in the subcortical nuclei region were occasionally taken from one or two slices below the slice displayed in the figure where it was better visualized.

\section{Statistical Analysis}

Data were analyzed using $3 \times 2$ mixed effects factorial analyses of covariance with repeated measures. Analyses were done on seven metabolites, using both absolute metabolite values and metabolite to creatine ratios from two brain regions. Separate analyses were done on each measure, and the primary results reported are not adjusted for multiple comparisons. Diagnostic group with three levels (depressed diabetic, diabetic control, healthy controls) was the between-groups factor, and hemisphere was a within-subject factor. Age and sex were included in all models as covariates. An unstructured covariance matrix was specified, allowing for unequal variances in the hemispheres. Follow-up contrasts of interest were performed using $t$-tests. Significance level was set at 0.05 . Pearson correlations were calculated to assess the relationship between hemoglobin A1c, CVRF, and CIRS and the metabolite concentrations measured.

\section{RESULTS}

We studied the absolute concentrations of NAA, choline, myo-inositol, glutamine, glutamate, and Glx corrected for atrophy. In our study, glutamate and glutamine concentra- tions in bilateral subcortical voxels of diabetic depressed subjects were significantly lower as compared to healthy controls (see Table 4, Figure 3). Myo-inositol was significantly increased in diabetic controls and depressed diabetic patients in frontal white matter as compared to healthy controls (see Table 5, Figure 4). In frontal white matter, there were no significant hemispheric differences in metabolite concentrations. In subcortical voxels, there was a significant difference between hemispheres $(\mathrm{F}=4.52$, $\mathrm{df}=1,59, p=0.04)$ with the decrease in Glx concentration found in depressed diabetic subjects being more pronounced in the left subcortical voxel $(\mathrm{F}=7.63, \mathrm{df}=2,59$, $p=0.001)$ as compared to the right subcortical voxel $(\mathrm{F}=5.69, \mathrm{df}=2,54, p=0.006)$. There were no significant differences seen in the other metabolites measured.

In addition to measuring absolute values corrected for CSF differences, we examined ratios of metabolites to creatine. Myo-inositol/creatine ratios, in frontal white matter as well as subcortical regions, were also significantly increased in diabetic controls and depressed diabetic patients $(\mathrm{F}=5.5, \mathrm{df}=2,127, p=0.005)$. However, while Glx/creatine ratios in subcortical regions did not show an overall statistical difference between all subject groups $(\mathrm{F}=3.07, \mathrm{df}=2,22, p=0.05)$, there was a significant decrease in Glx/creatine ratios in depressed diabetic subjects as compared to diabetic control subjects $(p=0.02)$. While there were no significant correlations between glycemic control as measured by hemoglobin Alc levels and the metabolites of interest (Glx and myo-inositol), in diabetic control subjects, myo-inositol 
concentrations in total frontal white matter had a significant positive correlation (Figure 5) with CVRF $(r=0.32$, $p<0.05)$. There was also a significant positive correlation between total subcortical voxel concentration of Glx and CIRS $(r=0.32, p<0.05)$ in diabetic control subjects.

\section{DISCUSSION}

The principal findings to emerge from our study were significantly lower levels of subcortical glutamate and glutamine in diabetic depressed patients. To our knowledge,

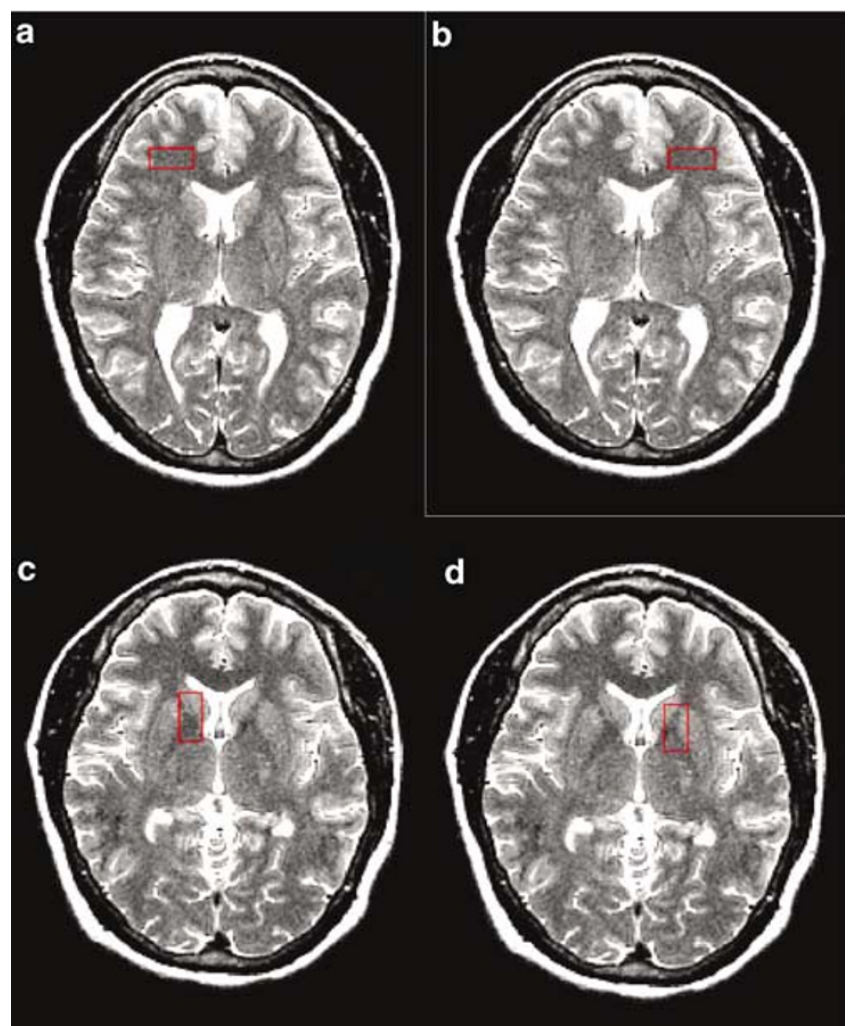

Figure 2 Voxel placement: anatomical locations of the voxels containing bilateral frontal white matter $(\mathrm{a}, \mathrm{b})$ and bilateral subcortical nuclei regions $(c, d)$. this is the first study to show such changes in subcortical regions. Most of the previous reports in the literature have shown these changes in cortical regions, particularly in the anterior cingulate (Auer et al, 2000; Rosenberg et al, 2005). We also demonstrated that myo-inositol levels were increased in the frontal white matter in diabetic patients with and without major depression.

A significant decrease in both glutamate and glutamine reflects what the literature has shown implicating the importance of glutamatergic transmission in mood disorders.

Previous studies using MRS to study the role of glutamate and glutamine in mood disorders have shown relatively consistent patterns of decreased glutamate in unipolar depression as opposed to an increase in glutamate associated with bipolar affective disorders. For example, MRS studies of major depression have demonstrated decreased Glx and glutamate concentrations in regions such as anterior cingulate (Auer et al, 2000; Rosenberg et al, 2005) and left dorsolateral prefrontal cortex (Michael et al, 2003a-c). However, children with bipolar affective disorder had higher glutamate/glutamine levels in frontal and

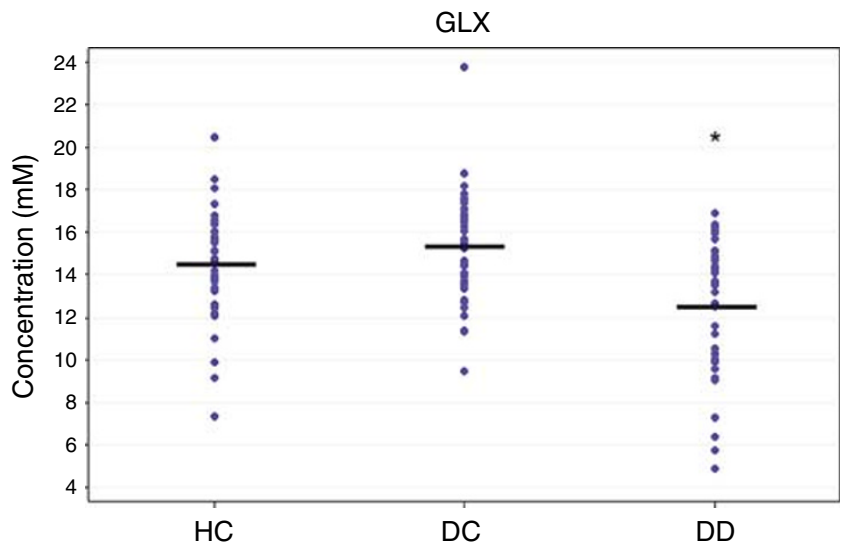

Figure $3 \mathrm{Glx}$ concentrations in bilateral subcortical voxels are significantly decreased in depressed patients; $* F=8.6, \quad d f=2,59$, $p=0.0005 ; \quad H C=$ healthy controls, $D C=$ diabetic controls, $D D=$ depressed diabetic subjects.

Table 4 Absolute Metabolite Concentrations in Subcortical Voxels

Subcortical voxels

\begin{tabular}{|c|c|c|c|c|c|c|}
\hline & \multicolumn{3}{|c|}{ Left } & \multicolumn{3}{|c|}{ Right } \\
\hline & HC & DC & DD & $\mathrm{HC}$ & DC & DD \\
\hline Choline & $1.49 \pm 0.23$ & $1.64 \pm 0.25$ & $1.43 \pm 0.26$ & $1.44 \pm 0.26$ & $1.46 \pm 0.17$ & $|.5| \pm 0.26$ \\
\hline Myo-Inositol & $4.18 \pm 1.14$ & $4.86 \pm 2.19$ & $4.23 \pm 1.23$ & $3.77 \pm 0.69$ & $4.79 \pm 1.79$ & $4.50 \pm 1.56$ \\
\hline Glutamine** & $5.97 \pm 2.75$ & $6.21 \pm 2.05$ & $4.60 \pm 2.08$ & $5.95 \pm 1.50$ & $5.59 \pm 2.02$ & $4.65 \pm 2.37$ \\
\hline
\end{tabular}

Values are millimolar concentrations shown as means $\pm \mathrm{SD}$

$* F=3.75, d f=2,59, p=0.03, * * F=4.68, d f=2,59, p=0.01, * * * F=8.6, d f=2,59, p=0.0005 ; H C$, healthy controls $(n=21)$; DC, diabetic controls $(n=24)$;

DD, depressed diabetic subjects $(n=20)$. 
Table 5 Absolute Metabolite Concentrations in Frontal White Matter

Frontal white matter

\begin{tabular}{|c|c|c|c|c|c|c|}
\hline & \multicolumn{3}{|c|}{ Left } & \multicolumn{3}{|c|}{ Right } \\
\hline & HC & DC & DD & HC & DC & DD \\
\hline Choline & $1.32 \pm 0.22$ & $1.47 \pm 0.33$ & $1.45 \pm 0.32$ & $1.36 \pm 0.29$ & $1.44 \pm 0.33$ & $1.43 \pm 0.25$ \\
\hline Myo-Inositol* & $4.03 \pm 1.21$ & $4.67 \pm 1.62$ & $4.69 \pm 1.38$ & $3.73 \pm 1.15$ & $4.66 \pm 1.07$ & $4.54 \pm 0.98$ \\
\hline Glutamine & $3.21 \pm 1.94$ & $3.39 \pm 2.06$ & $3.04 \pm 1.69$ & $3.20 \pm 1.73$ & $3.03 \pm 2.00$ & $3.05 \pm 1.58$ \\
\hline
\end{tabular}

Values are millimolar concentrations shown as means $\pm \mathrm{SD}$.

$* F=3.57, \mathrm{df}=2, p=0.03, \mathrm{HC}$, healthy controls $(n=21) ; \mathrm{DC}$, diabetic controls $(n=24)$; DD, depressed diabetic subjects $(n=20)$.

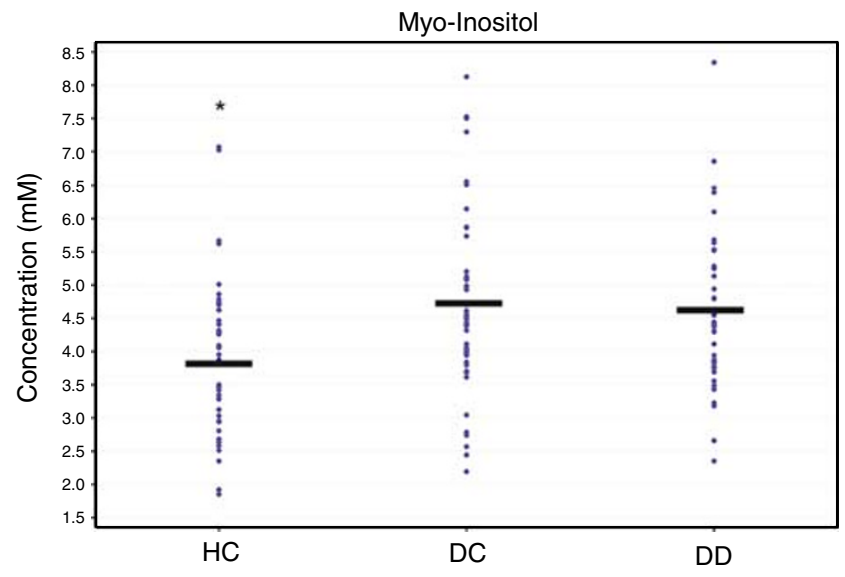

Figure 4 Myo-inositol concentrations in bilateral frontal white matter are significantly increased in diabetic patients with or without depression; ${ }^{*} \mathrm{~F}=3.57, \quad \mathrm{df}=2,59, \quad p=0.03 ; \quad \mathrm{HC}=$ healthy controls, $\mathrm{DC}=$ diabetic controls, $\mathrm{DD}=$ depressed diabetic subjects.

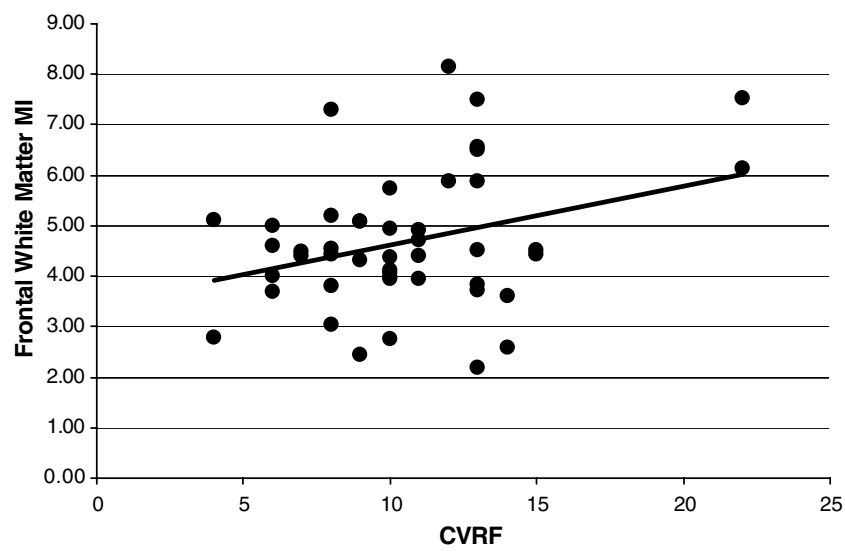

Figure 5 Significant positive correlation between frontal white $\mathrm{Ml}$ and CVRF in diabetic control subjects $(r=0.32, p<0.05)$.

subcortical regions when compared to controls in a study by Castillo et al (2000). It has also been shown that acute mania is associated with higher levels of Glx in left dorsolateral prefrontal cortex in adults with bipolar disorder (Michael et al, 2003a-c). The elevation of Glx has also been demonstrated in medication-free adult patients with bipolar disorder in recent study by Dager et al (2004).

Glutamate serves as an important excitatory neurotransmitter that can act in a functional role in synaptic plasticity crucial to learning and memory, as well as potential damaging agent involved in neuropathological conditions such as seizures, hypoxia, and hypoglycemia (Cooper et al, 1996). The pathological effect of glutamate is usually associated with excess levels of the metabolite, particularly in neurodegenerative diseases such as Alzheimer's disease (Lipton and Rosenberg, 1994). Selective decreases in glutamatergic function have been implicated in schizophrenia (Goff and Coyle, 2001). However, the implication of glutamate dysfunction in mood disorders is less well understood. In our study, the fact that both glutatamate and glutamine were significantly lower in depressed diabetics suggests that these subjects had lower precursor pools. Lower precursor pools may result from the combination of impaired glucose metabolism secondary to diabetes in conjunction with the perturbation of glial function associated with major depression. A model proposed by Sanacora et al (2003) described how impaired glial function could result in lower glutamate and glutamine levels in depressed patients. In their model, they distinguish synaptic glutamate levels from extrasynaptic concentrations and suggest that reduced glial function could lead to decreases in synaptic glutamate release and increases in extrasynaptic glutamate secondary to spillover. Data from the animal literature also suggests a role for the impact of diabetes on impaired glial function. Animal studies have shown altered glial metabolism in diabetic rats is necessary for the maintenance of glutamate and GABA concentrations (Garcia-Espinosa et al, 2003). This suggests that white matter alterations may be related to the differences in glutamate and glutamine concentrations seen in subcortical voxels.

Biochemical alterations in white matter were also demonstrated in our results. Myo-inositol was significantly increased in the frontal white matter of patients with diabetes with or without depression as compared to healthy control subjects. This is consistent with previous studies measuring brain metabolites in diabetic subjects. For 
example, increases in myo-inositol, glucose, and choline were seen in both gray and white matter in an early study of diabetic patients (Kreis and Ross, 1992). Also, myoinositol:creatine ratios have been shown to be increased in diabetic patients in both gray and white matter (Geissler et al, 2003). Myo-inositol increases have also been demonstrated in animal models of diabetes, albeit in hippocampus (van der Graaf et al, 2004). Most recently, myo-inositol increases in white matter were seen in diabetic patients with retinopathy and autonomic neuropathy (Makimattila et al, 2004). It has been suggested that the increases seen in myoinositol found in diabetic patients are associated with gliosis (Geissler et al, 2003). This hypothesis is consistent with our findings showing significant myo-inositol changes only in frontal white matter. In addition, there was a significant correlation between CVRF and frontal white matter myoinositol levels, which may suggest a vascular/ischemic basis to the gliosis in diabetes. There was no significant correlation between Hgb A1c levels and frontal white matter myo-inositol levels suggesting that the medical co-morbidities associated with diabetes are a more important factor than the degree of glycemic control in our study subjects. This is consistent with a study by Lyness et al (1998) where they showed an association between diabetes and late-life depression that lost statistical significance when medical disability was controlled for.

We did not find any significant differences in NAA and choline between our subjects. Based on previous studies, we expected to see decreased NAA and increased choline associated with impaired neuronal viability and white matter disease. Murata et al (2001) showed that lower levels of NAA:creatine ratios correlated significantly with the severity of white-matter disease. Work in our laboratory has shown significant increases in choline: creatine ratios and in myo-inositol:creatine ratios in the dorsolateral white matter of depressed patients as compared to controls (Kumar and Cook, 2002). This finding has been further explored looking at the relationship between brain biochemistry and white-matter disease. In normal-appearing white matter, magnetization transfer (MT) imaging, indicative of more subtle white-matter disease, was significantly correlated with changes in myo-inositol: creatine ratios and total NAA: creatine ratios measured in dorsolateral prefrontal cortex (Wyckoff et al, 2003).

In conclusion, the findings of this study demonstrate increased white matter myo-inositol is associated with type 2 diabetes and decreased subcortical glutamate/glutamine concentrations are associated with major depression in patients with type 2 diabetes. Given our interest in four specific metabolites, we did not correct for multiple comparisons, which would have reflected the measurement of seven metabolites in two hemispheres. To address certain limitations of the present study, future research will involve the addition of a major depression only group to determine to what extent the decrease in glutamate/glutamine is attributable to the combination of type 2 diabetes and depression $v s$ major depression alone. In addition, measurement of GABA in future studies will help to clarify possible mechanism underlying the differences demonstrated in glutamate concentrations. More specifically, to see if GABA levels are lower in subcortical voxels as a result of reduced glial function. These further studies are needed to relate our present results to the notion that microvascular disease that results from pathological conditions such as type 2 diabetes can cause disruptions in frontal-subcortical circuits that may underlie a putative physiological mechanism for major depression.

\section{ACKNOWLEDGEMENTS}

This research was funded by the National Institute of Health (R01-MH-63674, MH-55115, MH-61567, MH-02043) awarded to Anand Kumar, MD, the General Clinical Research Center (GCRC) and the UCLA Medical Center.

\section{REFERENCES}

Auer DP, Putz B, Kraft E, Lipinski B, Schill J, Holsboer F (2000). Reduced glutamate in the anterior cingulate cortex in depression: an in vivo proton magnetic resonance spectroscopy study. Biol Psychiatry 47: 305-313.

Castillo M, Kwock L, Courvoisie H, Hooper SR (2000). Proton MR spectroscopy in children with bipolar affective disorder: preliminary observations. AJNR Am J Neuroradiol 21: 832-838.

Centers for Disease Control and Prevention (2004). National diabetes fact sheet: general information and national estimates on diabetes in the United States 2003, Rev edn. US Department of Health and Human Services, Centers for Disease Control and Prevention: Atlanta, GA.

Cooper JR, Bloom FE, Roth RH (1996). The Biochemical Basis of Neuropharmacology. Oxford University Press, New York, 7th edn. pp 124-190.

Dager SR, Friedman SD, Parow A, Demopulos C, Stoll AL, Lyoo IK et al (2004). Brain metabolic alterations in medication-free patients with bipolar disorder. Arch Gen Psychiatry 61: 450-458.

D’Agostino RB, Wolf PA, Belanger AJ, Kannel WB (1994). Stroke risk profile: adjustment for antihypertensive medication. The Framingham Study. Stroke 25: 40-43.

de Groot M, Anderson R, Freedland KE, Clouse RE, Lustman PJ (2001). Association of depression and diabetes complications: a meta-analysis. Psychosom Med 63: 619-630.

Eaton WW, Armenian H, Gallo J, Pratt L, Ford DE (1996). Depression and risk for onset of type II diabetes. A prospective population-based study. Diabet Care 19: 1097-1102.

Folstein MF, Folstein SE, McHugh PR (1975). 'Mini-Mental State': a practical method for grading the cognitive state of patients for the clinician. J Psychiatr Res 12: 189-198.

Garcia-Espinosa MA, Garcia-Martin ML, Cerdan S (2003). Role of glial metabolism in diabetic encephalopathy as detected by high resolution 13C NMR. NMR Biomed 16: 440-449.

Gavard JA, Lustman PJ, Clouse RE (1993). Prevalence of depression in adults with diabetes. An epidemiological evaluation. Diabet Care 6: 1167-1178.

Geissler A, Frund R, Scholmerich J, Feuerbach S, Zietz B (2003). Alterations of cerebral metabolism in patients with diabetes mellitus studied by proton magnetic resonance spectroscopy. Exp Clin Endocrinol Diabet 111: 421-427.

Goff DC, Coyle JT (2001). The emerging role of glutamate in the pathophysiology and treatment of schizophrenia. Am J Psychiatry 158: $1367-1377$.

Hamilton M (1967). Development of a rating scale for primary depressive illness. Br J Soc Clin Psychol 6: 278-296.

Katon WJ, Lin EH, Russo J, Von Korff M, Ciechanowski P, Simon $\mathrm{G}$ et al (2004). Cardiac risk factors in patients with diabetes mellitus and major depression. J Gen Intern Med 19: 1192-1199.

Kollokia V (1996). Perfromance analysis of automatic techniques for tissue classification in magnetic resonance images of the 
human brain (master's thesis, 1996). Department of Computer Science, Concordia University, Montreal.

Kreis R, Ross BD (1992). Cerebral metabolic disturbances in patients with subacute and chronic diabetes mellitus: detection with proton MR spectroscopy. Radiology 184: 123-130.

Kumar A, Cook IA (2002). White matter injury, neural connectivity and the pathophysiology of psychiatric disorders. Dev Neurosci 24: 255-261.

Kumar A, Thomas A, Lavretsky H, Yue K, Huda A, Curran J et al (2002). Frontal white matter biochemical abnormalities in latelife major depression detected with proton magnetic resonance spectroscopy. Am J Psychiatry 159: 630-636.

Linn BS, Linn MW, Gurel L (1968). Cumulative illness rating scale. J Am Geriatr Soc 16: 622-626.

Lipton SA, Rosenberg PA (1994). Excitatory amino acids as a final common pathway for neurologic disorders. $N$ Engl J Med 330: 613-622.

Lustman PJ, Clouse RE (2005). Depression in diabetic patients: the relationship between mood and glycemic control. J Diabet Complicat 19: 113-122.

Lyness JM, Caine ED, Cox C, King DA, Conwell Y, Olivares T (1998). Cerebrovascular risk factors and later-life major depression. Testing a small-vessel brain disease model. Am J Geriatr Psychiatry 6: 5-13.

Makimattila S, Malmberg-Ceder K, Hakkinen AM, Vuori K, Salonen O, Summanen P et al (2004). Brain metabolic alterations in patients with type 1 diabetes-hyperglycemia-induced injury. J Cereb Blood Flow Metab 24: 1393-1399.

Mervaala E, Fohr J, Kononen M, Valkonen-Korhonen M, Vainio P, Partanen K et al (2000). Related Articles, Quantitative MRI of the hippocampus and amygdala in severe depression. Psychol Med 30: $117-125$.

Michael N, Erfurth A, Ohrmann P, Arolt V, Heindel W, Pfleiderer B (2003a). Neurotrophic effects of electroconvulsive therapy: a proton magnetic resonance study of the left amygdalar region in patients with treatment-resistant depression. Neuropsychopharmacology 28: 720-725.

Michael N, Erfurth A, Ohrmann P, Arolt V, Heindel W, Pfleiderer B (2003b). Metabolic changes within the left dorsolateral pre- frontal cortex occurring with electroconvulsive therapy in patients with treatment resistant unipolar depression. Psychol Med 33: 1277-1284.

Michael N, Erfurth A, Ohrmann P, Gossling M, Arolt V, Heindel W et al (2003c). Acute mania is accompanied by elevated glutamate/glutamine levels within the left dorsolateral prefrontal cortex. Psychopharmacology (Berlin) 168: 344-346.

Murata T, Kimura H, Omori M, Kado H, Kosaka H, Iidaka T et al (2001). MRI white matter hyperintensities, (1)H-MR spectroscopy and cognitive function in geriatric depression: a comparison of early- and late-onset cases. Int $J$ Geriatr Psychiatry 16: 1129-1135.

Palinkas LA, Lee PP, Barrett-Connor E (2004). A prospective study of Type 2 diabetes and depressive symptoms in the elderly: the Rancho Bernardo Study. Diabet Med 21: 1185-1191.

Rosenberg DR, Macmaster FP, Mirza Y, Smith JM, Easter PC, Banerjee SP et al (2005). Reduced anterior cingulate glutamate in pediatric major depression: A Magnetic Resonance Spectroscopy Study. Biol Psychiatry 58: 700-704.

Sanacora G, Gueorguieva R, Epperson CN, Wu YT, Appel M, Rothman DL et al (2004). Subtype-specific alterations of gammaaminobutyric acid and glutamate in patients with major depression. Arch Gen Psychiatry 61: 705-713.

Sanacora G, Rothman DL, Mason G, Krystal JH (2003). Clinical studies implementing glutamate neurotransmission in mood disorders. Ann NY Acad Sci 1: 292-308.

van der Graaf M, Janssen SW, van Asten JJ, Hermus AR, Sweep CG, Pikkemaat JA et al (2004). Metabolic profile of the hippocampus of Zucker Diabetic Fatty rats assessed by in vivo $1 \mathrm{H}$ magnetic resonance spectroscopy. NMR Biomed 17: 405-410.

Vythilingam M, Charles HC, Tupler LA, Blitchington T, Kelly L, Krishnan KR (2003). Focal and lateralized subcortical abnormalities in unipolar major depressive disorder: an automated multivoxel proton magnetic resonance spectroscopy study. Biol Psychiatry 54: 744-750.

Wyckoff N, Kumar A, Gupta RC, Alger J, Hwang S, Thomas MA (2003). Magnetization transfer imaging and magnetic resonance spectroscopy of normal-appearing white matter in late-life major depression. J Magn Reson Imaging 18: 537-543. 